\title{
Effect of carbon black on electrical and rheological properties of graphite nanoplatelets/poly(ethylene-butyl acrylate) composites
}

\author{
H. Oxfall ${ }^{1,2^{*}}$, G. Ariu ${ }^{1,3}$, T. Gkourmpis ${ }^{4}$, R. W. Rychwalski ${ }^{1}$, M. Rigdahl ${ }^{1}$ \\ ${ }^{1}$ Department of Materials and Manufacturing Technology, Chalmers University of Technology, SE-412 96 Gothenburg, \\ Sweden \\ ${ }^{2}$ Swerea IVF AB, SE-431 22 Mölndal, Sweden \\ ${ }^{3}$ Department of Materials Engineering and Industrial Technologies, University of Trento, I-38050 Trento, Italy \\ ${ }^{4}$ Borealis AB, Innovation \& Technology, SE-444 86 Stenungsund, Sweden
}

Received 27 May 2014; accepted in revised form 10 August 2014

\begin{abstract}
The effect of adding carbon black on the electrical and rheological properties of graphite nanoplatelets/poly(ethylene-butyl acrylate) copolymer composites produced via melt or solution mixing was studied. By adding a small amount of low- or high-structured carbon black to the nanocomposite, the electrical percolation threshold decreased and the final conductivity (at higher filler contents) increased. The effect on the percolation threshold was significantly stronger in case of the high-structured carbon black where replacing $10 \mathrm{wt} \%$ of the total filler content with carbon black instead of graphite nanoplatelets reduced the electrical percolation threshold from 6.9 to $4.6 \mathrm{vol} \%$. Finally, the solution mixing process was found to be more efficient leading to a lower percolation threshold. For the composites containing high-structured carbon black, graphite nanoplatelets and their hybrids there was a quite reasonable correlation between the electrical and rheological percolation thresholds.
\end{abstract}

Keywords: nanocomposites, hybrid composites, electrical properties, rheology, melt mixing

\section{Introduction}

Polymer nanocomposites combine the properties of the polymeric matrix with those of the filler. Traditionally carbon black (CB) has been the filler of choice due to the simplicity of its preparation methods that lead to low prices coupled with good overall performance [1]. The introduction of carbonaceous fillers into the polymer matrix can lead to electrical conductivity, whose level is tightly dependent on the type of filler and polymer used. Depending on the filler and the conductivity level required a large array of materials suited for electrochemical devices and other applications (e.g. fuel cells, batteries, supercapacitors, power cable shielding, auto- motive boards, chemical vapor sensors, pipe applications etc.) can be designed $[2,3]$. The conductivity level required for industrial applications varies, but can be quite high leading to an increase of the overall viscosity, thus leading to processing and performance problems [4].

High aspect ratio fillers based on carbon allotropes like graphene and carbon nanotubes (CNT) offer distinct advantages in comparison with more traditional fillers like carbon black mainly originating from their sheer size [5]. Ever since the first observations of graphene in 2004 [6], graphene-containing polymer nanocomposites have triggered a significant interest. A large number of research groups

\footnotetext{
${ }^{*}$ Corresponding author, e-mail: henrik.oxfall@gmail.com (C) BME-PT
} 
have published a variety of results indicating superior electrical and thermal conductivity, mechanical and rheological properties, gas barrier properties and crystallization behavior of nanocomposites based on high aspect ratio carbon allotropes [5, 7-9].

Hybrid composites of mixtures between different carbonaceous fillers have been seen to exhibit synergistic effects. Fan et al. [10] noted a decrease of the percolation threshold and a higher electrical conductivity by combining CB with graphite nanoplatelets (GNP). Similar positive effects have been observed in carbon nanotube (CNT)/graphite nanoplatelet hybrids [11-13] and CNT/GNP/CB hybrids [14]. Furthermore, by combining CNT with CB or GNP the viscosity of the composite melt has been reported to decrease in comparison to single filler composite melt at the same filler loading [15]. This effect was observed already at a content of $1 \mathrm{wt} \%$ CNT.

Published results on GNP-CB hybrids are often based on thermosetting polymer systems and highstructured carbon black. With the term high-structured $\mathrm{CB}$ we are referring to systems made by fusion of the primary particles into an extended three dimensional structure. The overall dimensions and density of this structure (it can be chain or cluster-like) depend on the preparation method of the CB [16]. There is a need to further explore possible synergistic effects of these hybrids in thermoplastic polymers and study the effect of the CB structure. In the present work, the effects of adding low- and highstructured carbon black, on electrical and rheological properties of a composite based on GNP/poly(ethylene-butyl acrylate) copolymer (EBA) are reported. Different mixing methods are employed with the solvent processing route used as reference. Electrical conductivity and rheological properties were measured along with characterization of the composites using differential scanning calorimetry (DSC) and scanning electron microscopy (SEM). Hybridization of carbon fillers is particularly applicable to extruded materials, where nanoplatelets tend to orient, leading to a need to create conductive paths/ bridges between the different filler particles as to maintain the integrity of the conductive network. Due to this fact and since extrusion is a major processing technique, the electrical conductivity measurements and the SEM studies were performed on extruded specimens.

\section{Experimental}

\subsection{Materials}

The polymer used was a poly(ethylene-butyl acrylate) random copolymer (EBA) with $17 \mathrm{wt} \%$ butyl acrylate (BA), MFR of $7 \mathrm{~g} / 10 \mathrm{~min}$ at $190^{\circ} \mathrm{C}$ and a density of $0.925 \mathrm{~g} / \mathrm{cm}^{3}$ (Borealis AB, Sweden).

A high-structured carbon black (HS-CB), Ketjenblack EC 600JD (AKZO Nobel, the Netherlands), with an aggregate size of $10-50 \mathrm{~nm}$, density $1.8 \mathrm{~g} / \mathrm{cm}^{3}$, BET surface area $1250 \mathrm{~m}^{2} / \mathrm{g}$, according to the supplier, was used. Also a low-structured furnace carbon black (LS-CB) with a surface area of $100-200 \mathrm{~m}^{2} / \mathrm{g}$ and a density of $1.8 \mathrm{~g} / \mathrm{cm}^{3}$ was used. The graphite nanoplatelets (GNP) used were $x \mathrm{GnP}$ M5 (XG Sciences, USA), with a thickness of 6-8 nm, diameter of $5 \mu \mathrm{m}$, density of $2.2 \mathrm{~g} / \mathrm{cm}^{3}$ and surface area of $120-150 \mathrm{~m}^{2} / \mathrm{g}$, as given by the manufacturer.

\subsection{Compounding}

A series of composites containing pure fillers and hybrids in which GNPs were combined with $\mathrm{CB}$ (both low- and high-structured) were manufactured. The hybrid compositions used were 90-10, 80-20 and 70-30 for the high-structured CB and 80-20, 70-30 and 60-40 for the low-structured CB. The first number in the hybrid composition denotes the wt $\%$ of GNP of the total filler content and the second the $w t \%$ of $\mathrm{CB}$. The filler contents used were in the range of $0.5-6.5$ volume- $\%$ (vol $\%$ ) for the HS-CB system, $5-14$ vol\% for the GNP systems, $2-9$ vol $\%$ for the HS-CB/GNP hybrids and $6-18$ vol $\%$ for the LS-CB materials and its hybrids.

A Brabender mixing chamber, with a volume of $50 \mathrm{~cm}^{3}$, was used to compound the composites. The mixing was performed at $180^{\circ} \mathrm{C}$ at $100 \mathrm{rpm}$ for 10 minutes.

Xylene (AnalaR Normapur, VWR Prolabo) was used in a solvent mixing technique. EBA and xylene, $1: 15$ weight concentration, were stirred at $120^{\circ} \mathrm{C}$ until the polymer was completely dissolved after 2 hours. GNP and xylene, 1:50 weight concentration, were first stirred at room temperature for 15 minutes, and then sonicated for $4 \mathrm{~h}$ in a Branson $1510 \mathrm{E}-\mathrm{MTH}$ bath $(70 \mathrm{~W})$, Branson Ultrasonic. Every 30 minutes the sonication was shortly stopped and the mixture was stirred for 1 minute. After sonication, the GNPxylene mixture was heated and added to the EBAxylene mixture and stirred for an additional $30 \mathrm{~min}$ - 
utes. After the stirring, the solution was poured into wide bowls in order to evaporate the solvent overnight followed by a final drying in a vacuum oven at $70^{\circ} \mathrm{C}$ for $12 \mathrm{~h}$. This mixing technique was only used for the high structured CB hybrids and the pure GNP at a filler content of $10 \mathrm{wt} \%$.

\subsection{Scanning electron microscopy (SEM)}

Scanning electron micrographs were obtained using a low vacuum JSM-6610 LV from Jeol, Japan, with an acceleration voltage of $8 \mathrm{kV}$ and a JSM-7800F from Jeol with an acceleration voltage of $3 \mathrm{kV}$. The specimens, taken from extruded strings, were ion polished, perpendicular to the flow direction, with a broad ion beam (Gatan Iliont model 693, USA) at $4 \mathrm{kV}$ at $-60^{\circ} \mathrm{C}$, in order to obtain a smooth surface for SEM studies.

\subsection{Differential scanning calorimetry (DSC)}

For the DSC measurements, a TG-DTA/DSC STA 449 F1 Jupiter, Netzsch-Gerätebau GmbH, Germany, equipped with a thermocouple Type K, was used. The melting behavior was recorded during heating from $30-200^{\circ} \mathrm{C}$ under argon atmosphere at a heating rate of $10^{\circ} \mathrm{C} / \mathrm{min}$. Specimens were taken from extruded strings, similar to the ones used for the electrical measurements.

\subsection{Electrical measurements}

Electrical measurements were performed using the two-point technique [17]. The specimens were extruded in a capillary rheometer, Ceast Rheoscope 1000 6742/00, Ceast SpA, Pianezza, Italy, using a die with a length/diameter $(L / D)$ ratio of $10 / 1$ $[\mathrm{mm} / \mathrm{mm}]$ at $170^{\circ} \mathrm{C}$. A low piston speed, $2 \mathrm{~mm} / \mathrm{min}$, corresponding to a shear rate of $24.3 \mathrm{~s}^{-1}$, as well as a higher piston speed, $20 \mathrm{~mm} / \mathrm{min}\left(243 \mathrm{~s}^{-1}\right)$ were used, but only two of the systems were subjected to the higher shear rate. The extrudates were then cryofractured in liquid nitrogen to a length, $l$, of $2.5 \mathrm{~cm}$ and the fracture surfaces were painted with conductive silver paint. A voltage was applied over the length of the extrudate and the current was measured with a Fluke 8846A Digital multimeter and the resistance, $R$, was evaluated. The diameter of the extrudates were measured using a digital caliper and the cross section area, $A$, was calculated. The electrical conductivity, $\sigma$, could then be obtained from Equation (1):
$\sigma=\frac{A}{R l}$

Three different voltage levels were used; $10 \mathrm{~V}$ for materials with a conductivity higher than $10^{-3} \mathrm{~S} / \mathrm{cm}$, $100 \mathrm{~V}$ for materials with a conductivity between $10^{-5}$ and $10^{-3} \mathrm{~S} / \mathrm{cm}$ and $300 \mathrm{~V}$ for conductivities lower than $10^{-5} \mathrm{~S} / \mathrm{cm}$. For each system, ten specimens were employed, and the average values are reported. The standard deviation was below $20 \%$ for materials with conductivities higher than $10^{-3} \mathrm{~S} / \mathrm{cm}$ and it then increased with decreasing conductivity. Since a two-point method was used one cannot fully exclude the existence of surface currents. This could to some extent affect the measured conductivity.

\subsection{Rheological measurements}

A stress-controlled rotational rheometer, Rheometrics SR200, was used at $180^{\circ} \mathrm{C}$ under $\mathrm{N}_{2}$ atmosphere for evaluating the viscoelastic properties of the materials. A parallel plate fixture was employed into which compression molded discs, produced in Bucher press in a frame-like mold at $180^{\circ} \mathrm{C}$ and a total melting time of 10 minutes, with a diameter of $25 \mathrm{~mm}$ and a thickness of $2 \mathrm{~mm}$ were inserted. All measurements started with a dynamic time sweep conducted at $0.5 \mathrm{~Hz}$ using a low stress until stabilization of the storage modulus $G^{\prime}$ was attained, as suggested by Kim and Macosko [18, 19]. Dynamic stress sweeps were conducted in order to determine the limit of the linear viscoelastic region and dynamic frequency sweeps were then performed well below this limit from $10 \mathrm{~Hz}$ down to $0.002 \mathrm{~Hz}$. For each system two or three measurements were performed and the average values are reported.

\section{Results and discussion}

\subsection{Scanning electron micrographs}

Figures 1, 2 and 3 show scanning electron micrographs of the extruded 80-20 hybrid based on GNP and HS-CB at a filler content $5.6 \mathrm{vol} \%$. The micrographs were taken close to the surface of the extrudate. In Figure 1 it is can be seen that needle-like GNP stacks were oriented in the flow direction and parallel to the surface of the extrudate. The existence of large needle-like agglomerates indicates poor mixing and that the effective surface area of the filler was decreased compared to the initial surface area. At a higher magnification, Figure 2, it is 
evident that large agglomerates consist of thinner GNP platelets while thinner platelets with a thickness smaller than $50 \mathrm{~nm}$ were present in the material. At even higher magnification, Figure 3, platelets with a thickness smaller than $20 \mathrm{~nm}$ can be observed. Also in Figures 2 and 3, HS-CB particles can be

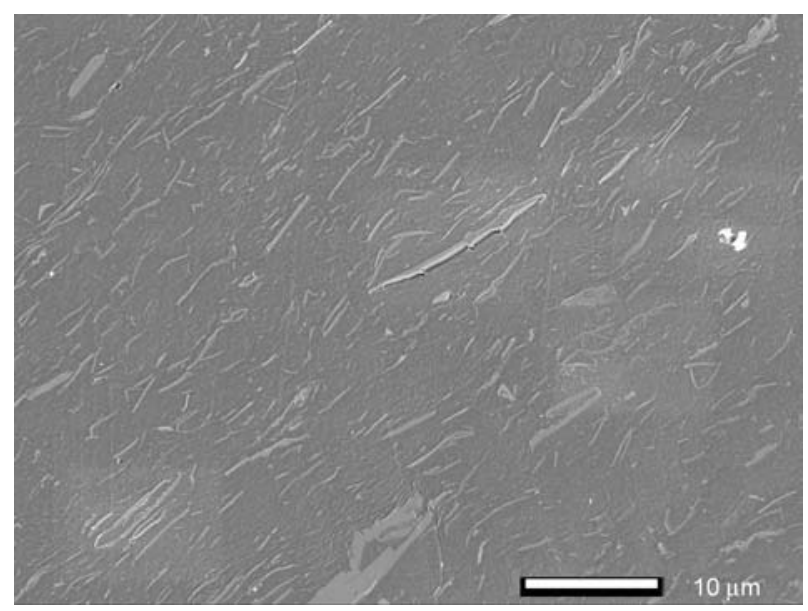

Figure 1. Scanning electron micrograph of the 80-20 GNP HS-CB hybrid at a magnification of $2000 \times$

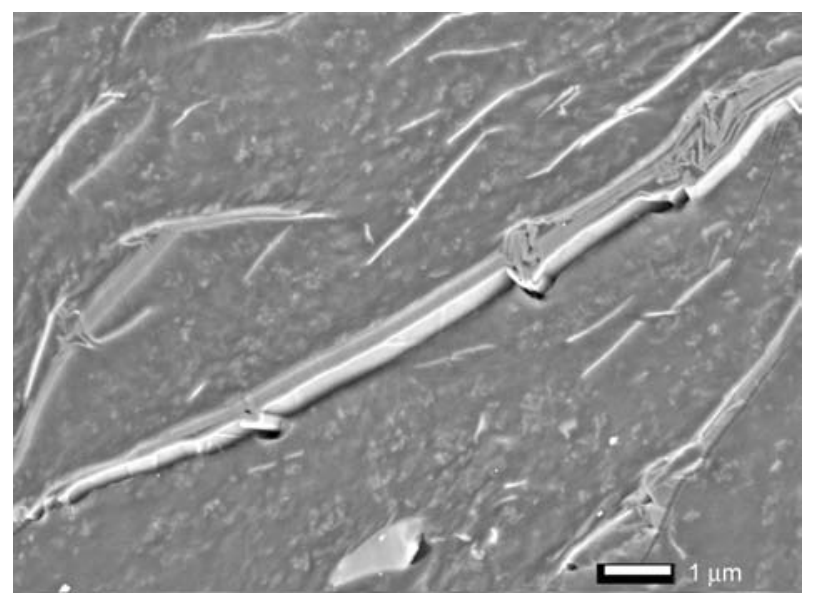

Figure 2. Scanning electron micrograph of the 80-20 GNP HS-CB hybrid at a magnification of $10000 \times$

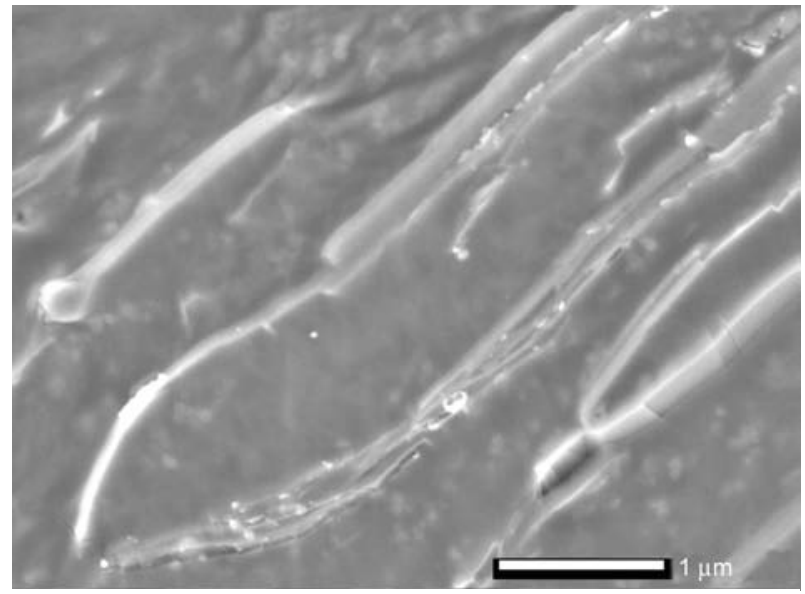

Figure 3. Scanning electron micrograph of the 80-20 GNP HS-CB hybrid at a magnification of $25000 \times$ observed between the GNP-stacks homogenously dispersed in the matrix. It is expected that the well dispersed CB particles could provide a physical bridge between the GNP stacks.

An identical orientation effect was observed also in the pure extruded GNP composite, both melt and solvent mixed. No clear differences could be observed with regard to the agglomerate size between the different mixing methods. In the HS-CB composite a good level of dispersion was noted with only few smaller agglomerates present.

\subsection{Thermal analysis}

The DSC measurements did not indicate clear differences in the crystallinity of the composites as compared to the pure polymer. There was a slight tendency towards higher crystallinity for the materials containing GNP and a slightly lower crystallinity for the materials containing $\mathrm{CB}$ compared to the unfilled EBA. A slightly narrower melting endotherm for the composite materials, especially the GNP composites, was observed and indicates that the crystals were thinner than in the unfilled EBA, se Figure 4. This can be attributed to the spatial arrangement of the filler particles that limit the possibility of polymer crystallization, as a number of nucleating sites become available for crystallization initiation. The existence of these different nucleation sites has been reported to lead to a decrease in crystallization rate $[20,21]$ The reason for such behavior has been attributed to the two-dimensional nature of the GNP platelets that makes lattice matching the dominant force in the crystallization process. The polymer chains need to be absorbed on the graphitic surface and this will force them to undergo conformational changes that subsequently lead to longer crystal-

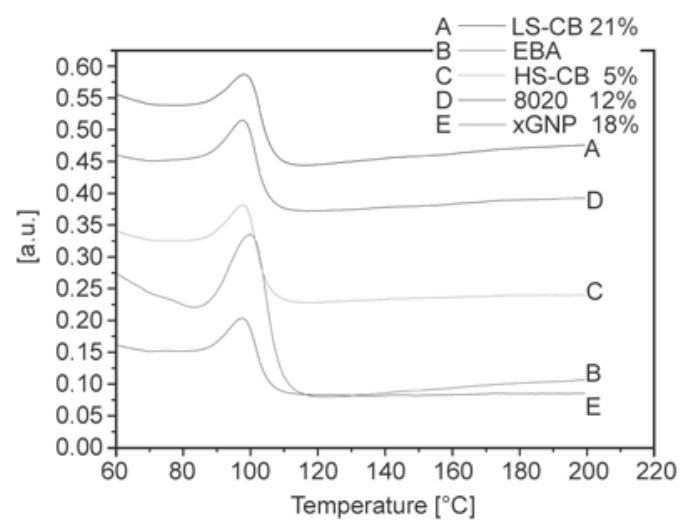

Figure 4. DSC thermograms for nanocomposites and matrix material 
lization times. Furthermore, due to the flat surface available for crystallization the polymer crystals can grow at multiple points and with multiple orientations. It has been speculated that the growth of adjacent crystals under these conditions might cause interference between them, leading to the overall suppression of the crystallization kinetics [21]. A similar behavior has been observed with GNP in polypropylene by Kalaitizidou et al. [22].

\subsection{Electrical conductivity}

Figure 5 shows the electrical conductivity as a function of the filler volume fraction for the GNP, the high-structured CB and its hybrids. The onset of electrical conductivity for the $\mathrm{CB}$ system was found to be the lowest, just below $2.1 \mathrm{vol} \%$, and the 'pure' GNP composite had the highest percolation threshold, just below 6.9 vol\%. The conductivity corresponding to the plateau value approached at higher filler contents was several orders of magnitude higher for the $\mathrm{CB}$ composites than for those containing GNP. In the work of Strååt et al. [23] the same kind of CB was mixed with both polypropylene (PP) and high density polyethylene (HDPE) and despite the fact that both processing and electrical measurements were conducted in a similar manner as in the present work, they report a lower percolation threshold for both polymer systems, being of the order $1 \mathrm{vol} \%$. The percolation threshold of $6.9 \mathrm{vol} \%$ (15 wt $\%$ ) found in this work for the pure melt mixed GNP composite is rather low compared to published results. Polyolefins containing GNP have usually a rather high percolation threshold, e. g. between 12 and $15 \mathrm{wt} \%$ in linear low density polyethylene and $14-16 \mathrm{wt} \%$ in an ethylene-vinyl acetate copolymer, both prepared by

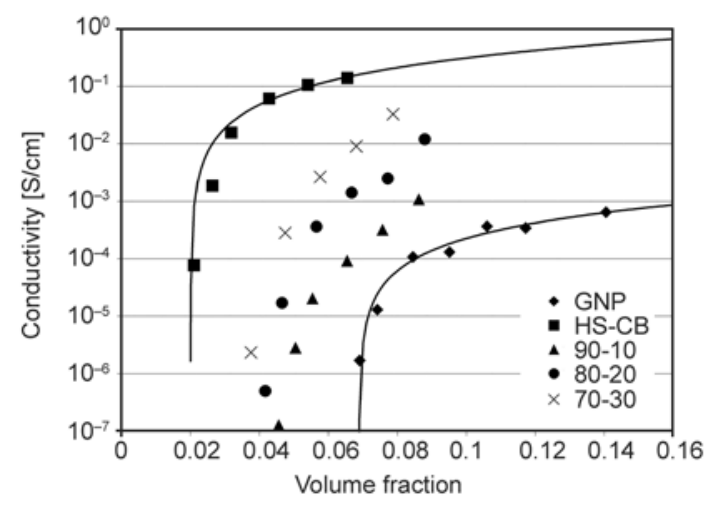

Figure 5. The electrical conductivity as a function of the filler content for composites containing GNP, HS$\mathrm{CB}$ and hybrids. Solid lines represent fitting to the percolation theory Equation (2). a solution mixing method which is more effective compared to melt-mixing [24, 25]. For polypropylene, a percolation threshold of $7 \mathrm{vol} \%$ has been found in a melt mixed material [26].

By replacing only $10 \mathrm{wt} \%$ of the total filler content with HS-CB instead of GNP, the electrical percolation was dramatically reduced, from 6.9 to $4.6 \mathrm{vol} \%$, whereas the conductivity plateau above the threshold increased (Figure 5). The same effect was observed when larger amounts of the overall filler content were replaced with CB. The effect of hybridization with CB has been studied earlier by Fan et al. [10] in epoxy-based composites, where by replacing $10 \%$ of the total filler content (GNP) with a high-structured carbon black the conductivity increased three orders of magnitude compared to the case with GNP alone when the total filler content was $1 \mathrm{wt} \%$. They also observed a decrease in the percolation threshold from 0.75 to $0.5 \mathrm{wt} \%$. This effect can be attributed to the existence of conductive bridges between the individual GNP agglomerates created by the CB particles. The reasoning for this is that high-structured CB being smaller and of significantly lower aspect ratio than GNP has a tendency of dispersing in a more efficient manner. This dispersion can act like a physical bridge between the GNP-rich areas in the matrix while at the same time restricts their dispersion and reagglomeration (see Figure 2). In the work by Wei et al. [14] carbon nanotubes were introduced in a similar system resulting in a three-fillerhybrid composite, and an even lower percolation threshold of $0.2 \mathrm{wt} \%$ was reported. These effects were also attributed to the ability of the spherical $\mathrm{CB}$ particles and the long flexible CNTs to form effective links between the more rigid GNPs.

The orientation in the flow direction of the anisometric fillers will influence the electrical properties. An extended level of orientation of the platelets could be expected to result in a higher percolation threshold due to a decreased particle-particle interaction $[5,9]$. Therefore it is expected that the extruded strands used in this study will exhibit a higher percolation threshold than the corresponding non-oriented composites. For the pure GNP system and the $80-20$ hybrid a higher piston speed $(20 \mathrm{~mm} / \mathrm{min})$ was used to enhance the orientation of the fillers. This increased the onset of electrical conductivity to approximately 7.4 and $4.7 \mathrm{vol} \%$, respectively, and can be compared with the results for $2 \mathrm{~mm} / \mathrm{min}$ where the onset was 6.9 and $4.2 \mathrm{vol} \%$, respectively. 
The experimental data was fitted to the classical percolation theory (Equation (2)):

$\sigma=\kappa\left(\omega-\omega_{\mathrm{c}}\right)^{\beta}$

where $\sigma$ is the conductivity of the composite, $\omega$ is the filler content, $\omega_{\mathrm{c}}$ the filler content at the percolation threshold, $\kappa$ and $\beta$ are fitting parameters where $\kappa$ is related to the conductivity of the filler and $\beta$ is connected to the systems dimensionality [27]. Here it must be noted that Equation (2) is derived for ideal conditions where all filler particles are of equal size, not agglomerated and non-oriented.

The percolation behavior of the pure CB and GNP systems could be fitted to Equation (2) as shown in Figure 5. The hybrid systems could not be fitted due to the lack of a clear conductivity plateau at higher loadings resulting in an unrealistic high value of $\kappa$. The lack of conductivity plateau is believed to originate from the increasing amount of $\mathrm{CB}$ with increasing filler content.

The conductivity results for the low-structured CB, the pure GNP and their hybrids are shown in Figure 6. The pure CB-based composite was seen to have a higher percolation threshold (12.1 vol\%) and conductivity plateau at high loadings than its GNP-based equivalent. In a similar study using poly(ethyl-vinyl acetate) copolymer as matrix, the higher level of the conductivity plateau for CB-based systems in comparison with the GNP-based ones was attributed to the extended agglomeration of the GNPs and the subsequent overall aspect ratio reduction [5]. The substantially higher percolation threshold of the LS-CB compared to the HS-CB is likely associated

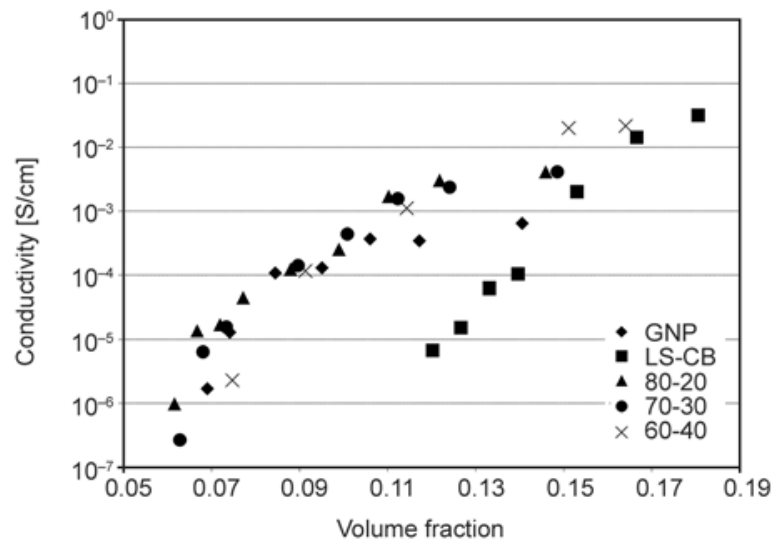

Figure 6. The electrical conductivity as a function of the filler content for composites containing GNP, LS$\mathrm{CB}$ and hybrids with the much lower BET surface area of the former particles.

For the low-structured CB hybrids, the percolation threshold decreased when some of the GNP was replaced by CB, at least in the case of the 80-20 and 70-30 hybrids. In the 60-40 hybrid the percolation threshold is slightly higher compared to the pure GNP but the conductivity plateau was, as for the other hybrids, higher than for the pure GNP system. This latter behavior can be discussed in a similar manner as for the HS-CB systems. Even though the percolation threshold was higher for the LS-CB compared to the GNP, the CB particles were effective in the building up a conductive network by connecting the 2-dimensional oriented GNPs with each other. A synergistic effect, not as strong as in the HS$\mathrm{CB}$ hybrids, was observed resulting in a decrease of the percolation threshold.

Figure 7 shows the electrical conductivity of the solvent-mixed materials as a function of the filler content. For the melt-mixed materials, the percolation threshold for the pure GNP systems was approximately $6.9 \mathrm{vol} \%$. Here it must be noted that the $10 \mathrm{wt} \%$ (4.5 vol\%) solution-mixed composition had similar conductivity as the $7.4 \mathrm{vol} \%$ melt-mixed composition indicating a significant decrease in the percolation threshold. The same pattern can be seen for the hybrid formulations where for example the solution-mixed $80-20$ hybrid at $10 \mathrm{wt} \%(4.7 \mathrm{vol} \%)$ had similar conductivity level as the 7.7 vol $\%$ melt mixed composition. Based on these results, the solution-mixing process was more efficient in terms of filler dispersion in comparison with the melt-mixing one for this specific combination of polymer

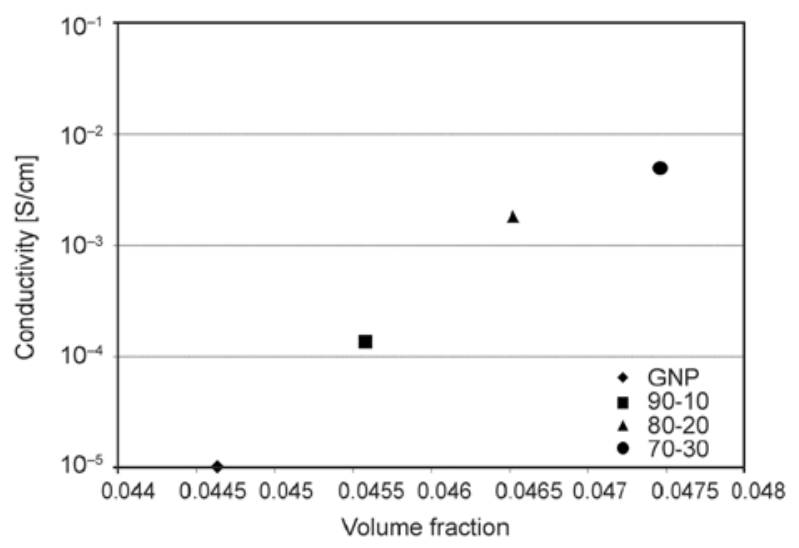

Figure 7. The electrical conductivity for solvent-mixed composites based on GNP or GNP/HS-CB containing $10 \mathrm{wt} \%$ filler 


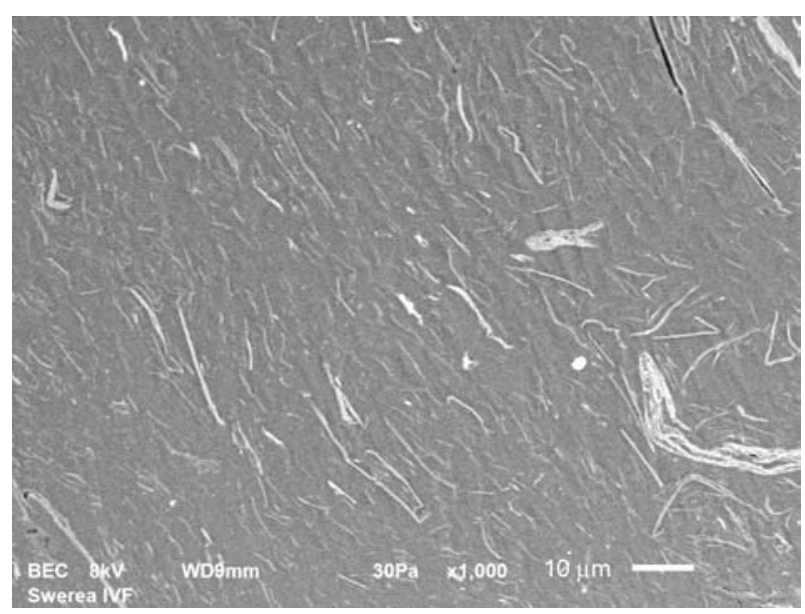

Figure 8. Scanning electron micrograph of the solventmixed material containing $10 \mathrm{wt} \% \mathrm{GNP}$ at a magnification of $1000 \times$

and filler, something that has been seen in earlier works of many authors [26, 28-30]. In the work by Oxfall et al., [28] GNP was mixed with polystyrene (PS), and it was observed that relatively large agglomerates were present in the composite after the melt mixing process. However when a solutionmixing method was employed, the number of large agglomerates was dramatically reduced resulting in a higher conductivity. In the present study, no differences could be discerned between the solvent and melt mixed systems with respect to the overall agglomerate size, se Figure 8. The decrease in percolation threshold observed when the solution-mixing method is used is thought to originate from the more efficient formation of thin graphite stacks [28]. Persson et al. [31] have shown that thin graphene stacks, consisting of mainly three layers and not more than five layers, are formed when employing solution mixing and these are likely to contribute to

Table 1. Electrical percolation thresholds for the different materials

\begin{tabular}{|l|c|c|}
\hline \multicolumn{1}{|c|}{ System } & $\begin{array}{c}\text { Percolation } \\
\text { threshold } \\
\text { [vol\%] }\end{array}$ & $\begin{array}{c}\text { Piston speed at } \\
\text { extrusion } \\
\text { [mm/min] }\end{array}$ \\
\hline GNP & $6.4<\omega_{\mathrm{c}}<6.9$ & 2 \\
\hline GNP & $6.9<\omega_{\mathrm{c}}<7.4$ & 20 \\
\hline HS-CB & $1.8<\omega_{\mathrm{c}}<2.1$ & 2 \\
\hline GNP/HS-CB, $90-10$ & $4.0<\omega_{\mathrm{c}}<4.6$ & 2 \\
\hline GNP/HS-CB, $80-20$ & $3.7<\omega_{\mathrm{c}}<4.2$ & 2 \\
\hline GNP/HS-CB, $80-20$ & $4.2<\omega_{\mathrm{c}}<4.7$ & 20 \\
\hline GNP/HS-CB, $70-30$ & $3.3<\omega_{\mathrm{c}}<3.8$ & 2 \\
\hline LS-CB & $11.4<\omega_{\mathrm{c}}<12.0$ & 2 \\
\hline GNP/LS-CB, $80-20$ & $5.6<\omega_{\mathrm{c}}<6.2$ & 2 \\
\hline GNP/LS-CB, $70-30$ & $5.8<\omega_{\mathrm{c}}<6.3$ & 2 \\
\hline GNP/LS-CB, $60-40$ & $6.9<\omega_{\mathrm{c}}<7.5$ & 2 \\
\hline
\end{tabular}

the lower percolation threshold in case of the equivalent systems observed in the present study.

Table 1 is a summary of the electrical percolation thresholds reported in this work and at which extrusion speeds the specimens were produced.

\subsection{Rheological properties}

Figures 9 and 10 show the storage modulus, $G^{\prime}$, as a function of frequency for the systems containing GNP, high-structured CB. Typically, there is a correlation between the plateau of $G^{\prime}$ at the lower frequencies (solid-like response) and the onset of the electrical conductivity (see references [5, 7,9 and 32] for further discussion). Another way of analyzing the rheological data is to find when $G^{\prime}$ becomes larger than the loss modulus $G^{\prime \prime}$ at low frequencies. This can also constitute a measure of the rheological percolation [33]. In the present work, the filler concentration where the cross-over between $G^{\prime}$ and $G^{\prime \prime}$

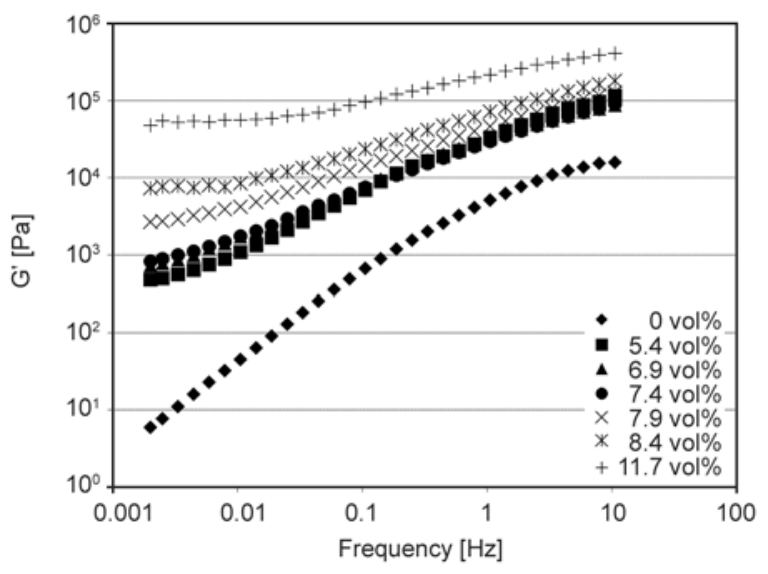

Figure 9. The storage modulus $G^{\prime}$ vs frequency for the GNP EBA composites at different filler contents measured at $180^{\circ} \mathrm{C}$

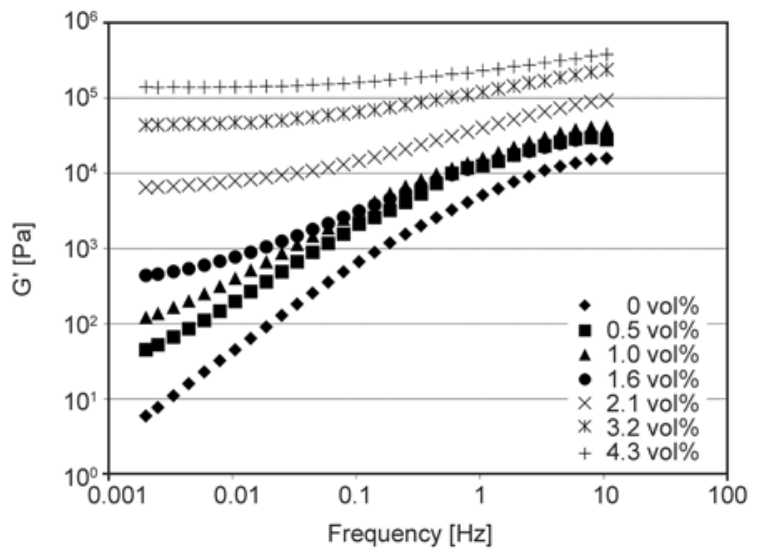

Figure 10. The storage modulus $G^{\prime}$ vs frequency for the HS-CB EBA composites at different filler contents measured at $180^{\circ} \mathrm{C}$ 


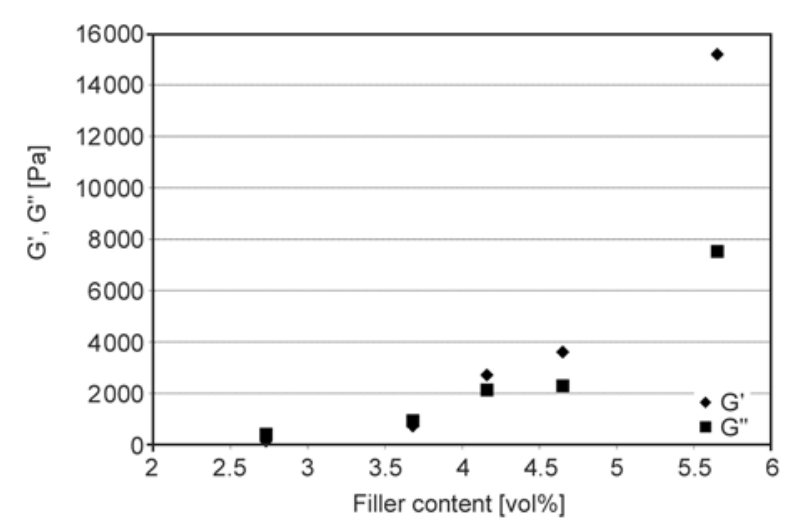

Figure 11. $G^{\prime}$ and $G^{\prime \prime}$ as a function of the filler content of the 80-20 hybrid containing GNP and HS-CB measured at $180^{\circ} \mathrm{C}$

took place was evaluated assuming a linear dependence of the moduli as functions of the filler content. The moduli $G^{\prime}$ and $G^{\prime \prime}$ as function of filler content for the 80-20 hybrid are shown in Figure 11. Table 2 shows the filler content at which the cross-over of the two moduli occurs. It should be made clear that an exact relation between the rheological and the electrical percolations are not expected in this case since the two types of test specimens differ in the degree of orientation; i.e. the rheological studies were conducted with non-oriented melts whereas the conductivity was measured using extruded strands. However similar trends can be noted when comparing the results in Tables 1 and 2.

The filler content at which the cross-over occurred was significantly higher for the GNP-based composite in comparison with the HS-CB-based system. As shown in Table 2, even small amounts of the highstructured $\mathrm{CB}$ reduced the cross-over concentration significantly in case of the HS-CB based hybrids. In the case of the LS-CB systems the rheological percolation (for the same filler content) was observed to be of similar order to the one observed for the GNP-composite. Replacing some of the GNP with

Table 2. The filler content at the $G^{\prime} / G^{\prime \prime}$ crossover (rheological percolation)

\begin{tabular}{|l|c|}
\hline \multicolumn{1}{|c|}{ System } & $\begin{array}{c}\mathbf{G}^{\prime} / \mathbf{G}^{\prime \prime} \text { cross over } \\
\text { [vol\%] }\end{array}$ \\
\hline GNP & 7.4 \\
\hline HS-CB & 1.5 \\
\hline GNP/HS-CB, 90-10 & 5.2 \\
\hline GNP/HS-CB, $80-20$ & 3.8 \\
\hline GNP/HS-CB, 70-30 & 3.2 \\
\hline LS-CB & 7.3 \\
\hline GNP/LS-CB, $80-20$ & 8.3 \\
\hline GNP/LS-CB, 70-30 & 8.6 \\
\hline GNP/LS-CB, 60-40 & 8.1 \\
\hline
\end{tabular}

the low structured CB actually produced an increase of the onset of rheological percolation.

Although it is not expected that there would be a very close agreement between the threshold values from the rheology and conductivity measurements, due to the orientation and possibly to the crystallization behavior, it is worthwhile to comment on this. First it can be noted that all composites exhibited a solid-like behavior at higher filler contents as manifested by the plateau in the storage modulus in the low frequency region. This can be associated with the formation of a filler network. The filler concentration at the $G^{\prime} / G^{\prime \prime}$ cross-over was higher for the GNP composite (no CB) than the electrical percolation threshold, (Tables 1 and 2). In Figure 9 it can be seen that the flattening of $G^{\prime}$ at lower frequencies took place between 7.4 and $7.9 \mathrm{vol} \%$ which is in correspondence with the $G^{\prime} / G^{\prime \prime}$ cross-over. In the case of the HS-CB, the $G^{\prime} / G^{\prime \prime}$ cross-over concentration was lower than the electrical percolation threshold. The cross-over concentration corresponded again reasonably well with the flattening of $G^{\prime}$ at $1.6 \mathrm{vol} \%$ (Figure 10). Based on this we can see that the GNP and the HS-CB systems behave in an opposite manner when the different percolation thresholds are compared.

With increasing amounts of HS-CB in the hybrid systems, the rheological threshold shifted from above the electrical threshold below it for the 70-30 hybrid. The correlation between the two thresholds is quite reasonable for the HS-CB/GNP systems considering the difference in orientation between the specimens. Strååt et al. [23] reported a good agreement between the electrical and the rheological thresholds in the case of PP and PE containing the same type of high-structured $\mathrm{CB}$ as used in this study. The conductivity measurements reported in this case [23] were also performed using extruded strands.

For the LS-CB systems the correlation between the rheological and the electrical percolation was clearly poorer. In Figure 12, $G^{\prime}$ as a function of frequency is shown for the LS-CB/EBA materials and it is evident that the flattening of $G^{\prime}$ at lower frequencies started already at $7.1 \mathrm{vol} \%$ compared to the onset of electrical conductivity between 11.4 and $12 \mathrm{vol} \%$. The $G^{\prime} / G^{\prime \prime}$ cross-over indicated a similar low value of $7.3 \mathrm{vol} \%$. The hybrids containing LS-CB exhibited, in similarity with the GNP/EBA material, a higher $G^{\prime} / G^{\prime \prime}$ cross-over compared to the 


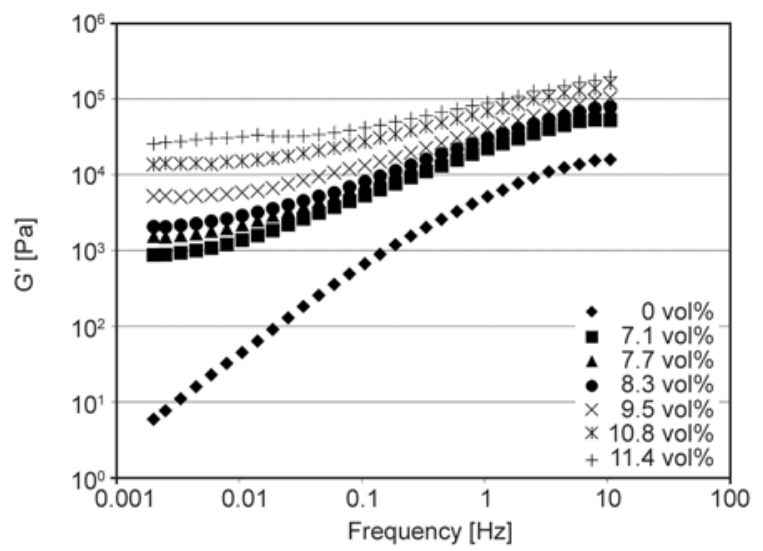

Figure 12. The storage modulus $G^{\prime}$ vs frequency for the LSCB EBA composites at different filler contents measured at $180^{\circ} \mathrm{C}$

electrical percolation. The difference between the two decreased however with increasing amounts of LS-CB, (Table 2).

\section{Conclusions}

The effect of both low- and high-structured carbon black on the electrical and the rheological properties of graphite nanoplatelet/poly(ethylene-butyl acrylate) composite have been assessed. It was found that the presence of both high- and low-structured $\mathrm{CB}$ in the matrix lead to a decrease of the electrical percolation threshold, with the effect being more pronounced in the case of the high-structured carbon black. This synergistic effect has been attributed to the creation of conductive bridges between the two different fillers.

In the extruded specimens used for electrical measurements a significant orientation of the filler particles in the flow direction was observed. The orientation had a significant effect on the overall conductivity of the composites.

Both melt and solution mixing methods were employed when producing the composite materials and the solution-based systems are seen to exhibit lower percolation threshold than the melt-mixed ones. This was attributed to the enhanced dispersion that is achieved by the solution process.

The presence of CB and GNP in the matrix affects the overall morphology of the system leading to thinner crystals and slight changes in the degree of crystallinity, evident by the slightly narrower melting endotherm and the shifted melting point.

Even though two types of test specimens that differed in the degree of orientation were used for the electrical and rheological measurements similar trends can be noted when comparing the electrical and rheological percolation thresholds. The composites containing high-structured carbon black, graphite nanoplatelets and their hybrids showed a quite reasonable correlation between the two types of percolation values. For the composites containing the low-structured carbon black, the correlation between the rheological and the electrical percolation thresholds was clearly poorer.

\section{Acknowledgements}

The authors thank Lars Eklund, Swerea IVF, for helping with the SEM studies, Christer Svanberg and Davide Tranchida, Borealis $\mathrm{AB}$, for valuable discussions. Finally we would like to thank the anonymous reviewers whose comments and suggestions greatly enhanced the manuscript. Financial support from the Swedish Foundation for Strategic Research (SSF) is gratefully acknowledged.

\section{References}

[1] Chodák I., Omastová M., Pionteck J.: Relation between electrical and mechanical properties of conducting polymer composites. Journal of Applied Polymer Science, 82, 1903-1906 (2001).

DOI: $10.1002 / a p p .2035$

[2] Lewis T. J.: Nanometric dielectrics. IEEE Transactions on Dielectrics and Electrical Insulation, 1, 812-825 (1994).

DOI: $10.1109 / 94.326653$

[3] Tanaka T., Montanari G. C., Mülhaupt R.: Polymer nanocomposites as dielectrics and electrical insulation-perspectives for processing technologies, material characterization and future applications. IEEE Transactions on Dielectrics and Electrical Insulation, 11, 763-784 (2004).

DOI: 10.1109/TDEI.2004.1349782

[4] Jeong K-U., Lim J. Y., Lee J-Y., Kang S. L., Nah C.: Polymer nanocomposites reinforced with multi-walled carbon nanotubes for semiconducting layers of highvoltage power cables. Polymer International, 59, 100106 (2010).

DOI: $10.1002 /$ pi.2696

[5] Gkourmpis T.: Carbon-based high aspect ratio polymer nanocomposites. in 'Nanoscience and computational chemistry: Research progress' (eds.: Mercader G. A., Castro E. A., Haghi A. K.) Apple Academic Press, Toronto, Vol 1, 85-124 (2014).

[6] Novoselov K. S., Geim A. K., Morozov S. V., Jiang D., Zhang Y., Dubonos S. V., Grigorieva I. V., Firsov A. A.: Electric field effect in atomically thin carbon films. Science, 306, 666-669 (2004).

DOI: $10.1126 /$ science. 1102896

[7] Potts J. R., Dreyer D. R., Bielawski C. W., Ruoff R. S.: Graphene-based polymer nanocomposites. Polymer, 52, 5-25 (2011). DOI: $10.1016 /$ j.polymer.2010.11.042 
[8] Sengupta R., Bhattacharya M., Bandyopadhyay S., Bhowmick A. K.: A review on the mechanical and electrical properties of graphite and modified graphite reinforced polymer composites. Progress in Polymer Science, 36, 638-670 (2011).

DOI: $10.1016 /$ j.progpolymsci.2010.11.003

[9] Kim H., Abdala A. A., Macosko C. W.: Graphene/polymer nanocomposites. Macromolecules, 43, 6515-6530 (2010).

DOI: $10.1021 / \mathrm{ma100572 \textrm {e }}$

[10] Fan Z., Zheng C., Wei T., Zhang Y., Luo G.: Effect of carbon black on electrical property of graphite nanoplatelets/epoxy resin composites. Polymer Engineering and Science, 49, 2041-2045 (2009).

DOI: 10.1002/pen.21445

[11] Ren P-G., Di Y-Y., Zhang Q., Li L., Pang H., Li Z-M.: Composites of ultrahigh-molecular-weight polyethylene with graphene sheets and/or MWCNTs with segregated network structure: Preparation and properties. Macromolecular Materials and Engineering, 297, 437443 (2012). DOI: $10.1002 /$ mame. 201100229

[12] Li J., Wong P-S., Kim J-K.: Hybrid nanocomposites containing carbon nanotubes and graphite nanoplatelets. Materials Science and Engineering: A, 483-484, 660663 (2008).

DOI: $10.1016 /$ j.msea.2006.08.145

[13] Kumar S., Sun L. L., Caceres S., Li B., Wood W., Perugini A., Maguire R. G., Zhong W. H.: Dynamic synergy of graphitic nanoplatelets and multi-walled carbon nanotubes in polyetherimide nanocomposites. Nanotechnology, 21, 105702/1-105702/10 (2010). DOI: $10.1088 / 0957-4484 / 21 / 10 / 105702$

[14] Wei T., Song L., Zheng C., Wang K., Yan J., Shao B., Fan Z-J.: The synergy of a three filler combination in the conductivity of epoxy composites. Materials Letters, 64, 2376-2379 (2010). DOI: $10.1016 /$ j.matlet.2010.07.061

[15] Via M. D., Morrison F. A., King J. A., Beach E. A., Wiese K. R., Bogucki G. R.: Effects of multiple carbon fillers on the rheology of polycarbonate-based composites. Polymer Composites, 33, 306-316 (2012). DOI: $10.1002 /$ pc.22149

[16] Hess W. M., Herd C. R.: Microstructure, morphology and general physical properties. in 'Carbon black science and technology’ (eds.: Donnet J-B., Bansal R. C., Wang M-J.) Taylor and Francis, London, 89-160 (1993).

[17] Heaney M. B.: Electrical conductivity and resistivity. in 'Electrical Measurement, Signal Processing and Displays' (ed.: Webster J. G.) CRC Press, Vol 1, 7/1-7/14 (2003).

[18] Kim H., Macosko C. W.: Processing-property relationships of polycarbonate/graphene composites. Polymer, 50, 3797-3809 (2009).

DOI: $10.1016 /$ j.polymer.2009.05.038
[19] Kim H., Macosko C. W.: Morphology and properties of polyester/exfoliated graphite nanocomposites. Macromolecules, 41, 3317-3327 (2008).

DOI: $10.1021 / \mathrm{ma} 702385 \mathrm{~h}$

[20] Xu J-Z., Chen T., Yang C-L., Li Z-M., Mao Y-M., Zeng B-Q., Hsiao B. S.: Isothermal crystallization of poly(L-lactide) induced by graphene nanosheets and carbon nanotubes: A comparative study. Macromolecules, 43, 5000-5008 (2010).

DOI: $10.1021 / \mathrm{ma100304n}$

[21] Xu J-Z., Chen C., Wang Y., Tang H., Li Z-M., Hsiao B. S.: Graphene nanosheets and shear flow induced crystallization in isotactic polypropylene nanocomposites. Macromolecules, 44, 2808-2818 (2011).

DOI: $10.1021 / \mathrm{ma} 1028104$

[22] Kalaitzidou K., Fukushima H., Askeland P., Drzal L. T.: The nucleating effect of exfoliated graphite nanoplatelets and their influence on the crystal structure and electrical conductivity of polypropylene nanocomposites. Journal of Materials Science, 43, 2895-2907 (2008). DOI: $10.1007 / \mathrm{s} 10853-007-1876-3$

[23] Strååt M., Toll S., Boldizar A., Rigdahl M., Hagström B.: Melt spinning of conducting polymeric composites containing carbonaceous fillers. Journal of Applied Polymer Science, 119, 3264-3272 (2011).

DOI: 10.1002/app.32882

[24] Kim S., Do I., Drzal L. T.: Multifunctional xGnP/ LLDPE nanocomposites prepared by solution compounding using various screw rotating systems. Macromolecular Materials and Engineering, 294, 196-205 (2009).

DOI: $10.1002 /$ mame.200800319

[25] Kim S., Drzal L. T.: Comparison of exfoliated graphite nanoplatelets $(\mathrm{xGnP})$ and $\mathrm{CNTs}$ for reinforcement of EVA nanocomposites fabricated by solution compounding method and three screw rotating systems. Journal of Adhesion Science and Technology, 23, 1623-1638 (2009). DOI: $10.1163 / 156856109 X 440984$

[26] Kalaitzidou K., Fukushima H., Drzal L. T.: A new compounding method for exfoliated graphite-polypropylene nanocomposites with enhanced flexural properties and lower percolation threshold. Composites Science and Technology, 67, 2045-2051 (2007).

DOI: 10.1016/j.compscitech.2006.11.014

[27] Bauhofer W., Kovacs J. Z.: A review and analysis of electrical percolation in carbon nanotube polymer composites. Composites Science and Technology, 69, 14861498 (2009).

DOI: 10.1016/j.compscitech.2008.06.018

[28] Oxfall H., Rondin J., Bouquey M., Muller R., Rigdahl M., Rychwalski R. W.: Elongational flow mixing for manufacturing of graphite nanoplatelet/polystyrene composites. Journal of Applied Polymer Science, 128, 2679-2686 (2013).

DOI: 10.1002/app.38439 
[29] Shen J-W., Chen X-M., Huang W-Y.: Structure and electrical properties of grafted polypropylene/graphite nanocomposites prepared by solution intercalation. Journal of Applied Polymer Science, 88, 1864-1869 (2003).

DOI: $10.1002 / a p p .11892$

[30] Kim H., Macosko C.: Morphology and properties of exfoliated graphite/thermoplastic polyurethane nanocomposites. in '2009 Annual Technical Conference ANTEC Conference Proceedings'. Chicago, USA, Vol 1, 122-125 (2009).
[31] Persson H., Yao Y., Klement U., Rychwalski R. W.: A simple way of improving graphite nanoplatelets (GNP) for their incorporation into a polymer matrix. Express Polymer Letters, 6, 142-147 (2012).

DOI: $10.3144 /$ expresspolymlett.2012.15

[32] Alig I., Pötschke P., Lellinger D., Skipa T., Pegel S., Kasaliwal G. R., Villmow T.: Establishment, morphology and properties of carbon nanotube networks in polymer melts. Polymer, 53, 4-28 (2012).

DOI: 10.1016/j.polymer.2011.10.063

[33] Kotsilkova R.: Thermoset nanocomposites for engineering applications. Rapra, Shawbury (2007). 